\title{
Potassium channel Origami windmill Model
}

\author{
Zuodong Sun * \\ Ya'ou Brain Science Institute of Heilongjiang province, Harbin, 150090, China
}

\begin{abstract}
The potassium channel model proposed in this paper is an independent functional unit, four $\alpha$-helixs rotate synchronously in one direction, it transports $\mathrm{K}^{+}$passively and unilaterally and has no dependence on ATP, that is different from previous models such as "paddle model" "propeller model " and "revolving door model ". Its mechanism is that $\mathrm{K}^{+}$ and the positively charged amino acids in $\alpha$-helixs form a repulsive force, which pushes the "blade" back and makes the "windmill" rotate. The aperture size of $\mathrm{K}^{+}$channel varies with the speed of windmill. This determines the "opening" and "closing" of channel holes. The model of "origami windmill" reveals the mechanism of $\mathrm{K}^{+}$channel operation by applying the principles of cell biophysics. This has enlightening significance for other basic research related to it. Perhaps, this will help answer the basic biological questions of human health and disease from the source.
\end{abstract}

Keywords: $\mathrm{K}^{+}$Channel; open; close; Origami Windmill; model; $\mathrm{K}^{+}$Transshipment

\footnotetext{
*Corresponding author: Zuodong Sun (E-mail: sunzuodong@pai314.com).
} 
In 2003, MacKinnon Laboratory According to three-dimensional Crystal Structure of Voltagegated KvAP Channel Protein, on the basis of Electrical-mechanical coupling, they proposed the " paddle model ", a kind of membrane potentiometric receptor. This model was later considered unreasonable $^{[1]}$. In 2005, MacKinnon's laboratory has again successfully analyzed the mammalian Kv1.2 potassium channel. They revised and supplemented the " paddle model" ${ }^{[2,3]}$. However, how Kv1.2 membrane potentiometric receptors are converted from "open" to "closed" remains unclear.

In this paper, we only discuss how $\mathrm{K}^{+}$of voltage-gated $\mathrm{K}^{+}$channel is transferred from extracellular membrane to intracellular, and the process and mechanism of $\mathrm{K}^{+}$channel "open" and "close". The proposed $\mathrm{K}^{+}$channel structure model is similar to the "origami windmill". This is based on the principle of cell biophysics and the observation of $\mathrm{K}^{+}$channel crystal structure in MacKinnon Laboratory.

Looking from the outside of the cell, the model looks very much like a rotating wheel with four blades. Each blades has an "open petal" ${ }^{[4,5]}, \alpha$ helix(Figure A). Four blades are like an origami windmill, its channel holes are formed by $\alpha$ helix. The four inner spirals overlap or entangle with each other near the inner part of the cell membrane. That forms an inverted cone structure. Its pore area is contained in the "petal" ${ }^{\text {"[6] }}$ (Figure B).

The origami windmill is an independent functional unit consisting of four alpha spirals, it is a rotating body, the rotating body is like an inverted cone, it is suspended in the lipid bilayer and rotates unilaterally. The repulsive force of extracellular cations exerts a limiting effect on its axis; The mode of transshipment of $\mathrm{K}^{+}$is passive, extracellular $\mathrm{K}^{+}$is "screwed" into cells. It only enters and not comes out. It has no dependence on ATP and it is also different from the "revolving door" and "propeller" model. "Rotary door" is bidirectional rotation, and it can not reasonably explain the $\mathrm{K}^{+}$in or out. And the "propeller" model needs a motor. "Cell" has the same meaning as battery, the function of origami windmill is equivalent to charging the battery. Generators and Motors are fundamentally different.

The operating principle of "origami windmill" model is well understood (Figures C and D). Negatively charged amino acid residues at $\mathrm{K}^{+}$channel produce a weak attraction to cations ${ }^{[6]}$. It attracts nearby cations Including $\mathrm{K}^{+}$, making them close to the $\alpha$ helix. It is known that the S4 helix contains many positively charged amino acids ${ }^{[7,8]} \cdot \mathrm{K}^{+}$and positively charged amino acids in the $\alpha$ helix form a repulsive force, which pushes the "blades" back and makes the "windmill" rotate. With the increase of $\mathrm{K}^{+}$at the entrance of the passage, the speed of windmill rotation "changes from slow to fast". Rotation makes the alpha helix extension outward, expands the scope of "capturing" $\mathrm{K}^{+}$and expands the channel aperture to a diameter larger than $\mathrm{K}^{+}$. The 
extracellular $\mathrm{K}^{+}$is "screwed" into cells in large quantities and accumulates on the inner surface of cell membranes. At this time, this is equivalent to the complete opening of the channel hole. When the concentration of $\mathrm{K}^{+}$and other cations near the adventitia decreases, the rotation speed of the windmill changes from fast to slow. The folded and contracted $\alpha$ helix extrudes the channel aperture to less than $\mathrm{K}^{+}$diameter, which can now be regarded as the channel aperture completely closed.

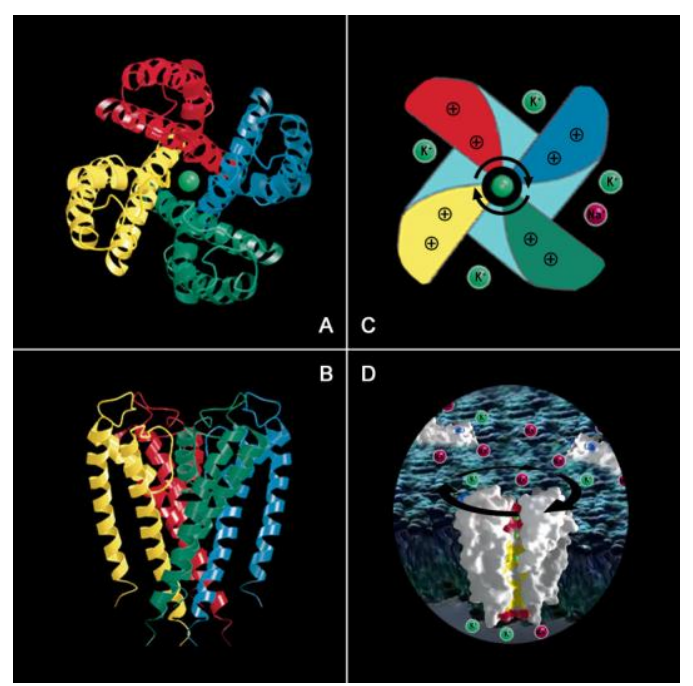

Figure A: Looking from the outside of the cell membrane, Three-dimensional strip map of KcsA potassium channel. Four subunits are marked by different colors, respectively(Quoted from Doyle et al. Science,1998). Figure B: Three-dimensional strip map of KcsA potassium channel obtained from the direction perpendicular to graph A(Quoted from Doyle et al.Science,1998). Figure C: Model sketch of origami windmill. Figure D: Stereo sectional view and rotation diagram of potassium channel surface.

MacKinnon Laboratory's observation results of the channel crystal structure support the "origami windmill" model theory. KcsA potassium channel is the structure when the channel hole is "closed". Its inner lining alpha helix forms a cross near the inner surface of the cell membrane. At the intersection, the channel aperture becomes narrower (less than $3.5 \AA$ ), which hinders $\mathrm{K}^{+}$flow. MthK Potassium channel is the structure when the channel hole is "opened". Its lining alpha helix bends and extends at the turning point, which enlarges the pore size of the channel (larger than $3.5 \AA$ ), and $\mathrm{K}^{+}$enters the cell smoothly ${ }^{[2,3,9]}$.

The model of "origami windmill" is falsifiable. Researchers have grafted propellers made of ferromagnetic nickel onto the mid-axis of adenosine triphosphate molecule. When they were immersed in ATP solution, five molecular motors turned $(8 \mathrm{r} / \mathrm{s})^{[10]}$. If the material of propeller is replaced by non-magnetic material, the molecular motor can still rotate. It can prove that the $\mathrm{K}^{+}$ channel "origami windmill" model is not existenced. The rotational behavior of DNA has been 
observed by scientific research teams ${ }^{[11]}$. Therefore, the verification of origami windmill model can be realized in the near future.

The proposition of "origami windmill" model reveals the operating mechanism of $\mathrm{K}^{+}$channel by applying the principles of physics. It has enlightening significance for some basic research related to it. It may help to answer the basic biological questions of human health and disease from the source. The application of this model principle can reasonably explain the whole process of resting potential and action potential, which will be published separately.

Author has been engaged in the research and development of basic theory of brain science and rehabilitation treatment equipment for encephalopathy since 1994. He has been supported by various national, provincial and municipal science and technology funds for many times. He has won the first prize of Heilongjiang Science and Technology Award (Invention) and Heilongjiang Excellent Youth Science Fund. Established in 2001, Ya'ou Brain Science Institute of Heilongjiang province in Asia and Europe is an independent legal entity and a professional academic research institution supported by Harbin Obo Medical Devices Co., Ltd. This Institute focuses on the basic theory of brain science.

\section{References}

[1] Jiang Y X, Lee A, Chen J, et al. X-ray structure of a voltage-dependent K+ channel[J]. Nature, 2003, 423(6935): 33-41.

[2] Long S B, Campbell E B, Mackinnon R. Crystal structure of a mammalian voltage-dependent shaker family K+ channel[J]. Science, 2005, 309(5736):897-903.

[3] Long S B, Campbell E B, Mackinnon R. Voltage sensor of Kv1.2: structural basis of electromechanical coupling[J]. Science, 2005, 309(5736):903-908.

[4] Heginbotham L, Abramson T, Mackinnon R. A functional connection between the pores of distantly related ion channels as revealed by mutant $\mathrm{K}+$ channels[J]. Science, 1992, 258(5085):1152-1155.

[5] Heginbotham L, Lu Z, Abramson T, et al. Mutations in the K+ channel signature sequence[J]. Biophysical Journal, 1994, 66(4):1061-1067.

[6] Doyle D A, Cabral J M, Pfuetzner R A, et al. The Structure of the Potassium Channel: Molecular Basis of K+ Conduction and Selectivity[J]. Science, 1998, 280: 69-77.

[7] Long S B, Tao X, Campbell E B, et al. Atomic structure of a voltage-dependent K+ channel in a lipid membrane-like environment[J]. Nature, 2007, 450(7168): 376-382.

[8] Parsegian V A. Ion-membrane interactions as structural forces[J]. Ann N Y Acad Sci, 1975, 264(Dec30): 161-174.

[9] Song F J, Guo J T. Progress on structural biology of voltage-gated ion channels[J]. Journal of Zhejiang University(Medical Sciences), 2019, 2:25-33.

[10] Shu Y G, OuYang Z C. Biological molecular motors[J]. Journal of Physics, 2007, 36(10):735-741.

[11] Pallav K, Benjamin D A, Mingjie D, et al. Rotation tracking of genome-processing enzymes using DNA origami rotors[J]. Nature, 2019, DOI: 10.1038/s41586-019-1397-7. 